Urologe $2021 \cdot 60: 1-2$

https://doi.org/10.1007/s00120-020-01417-0

Angenommen: 24. November 2020

(c) Springer Medizin Verlag $\mathrm{GmbH}$, ein Teil von Springer Nature 2020

\author{
Arkadiusz Miernik ${ }^{1}$ Thorsten Schlomm ${ }^{2} \cdot$ Christian Bolenz $^{3}$ \\ 'Medizinische Fakultät, Klinik für Urologie, Universitätsklinikum Freiburg, Freiburg, Deutschland \\ ${ }^{2}$ Klinik für Urologie, Charité - Universitätsmedizin Berlin, Berlin, Deutschland \\ ${ }^{3}$ Klinik für Urologie und Kinderurologie, Universitätsklinikum Ulm, Ulm, Deutschland
}

\title{
Innovationen in der Urologie: Garanten für den Fortschritt
}

Sehr geehrte Leserinnen und Leser, liebe Kolleginnen und Kollegen,

die hohe Motivation, den Status quo durch neue Diagnose- und Therapieverfahren zu verbessern, haben die Urologie seit jeher geprägt. Der medizinische Fortschritt kam unseren Patienten stets zugute - sowohl im Grundlagenbereich als auch bei translationalen Ansätzen.

Insbesondere an akademischen Einrichtungen werden zahlreiche Forschungsvorhaben durchgeführt und deren Ergebnisse publiziert. Nur wenige "Innovationen" schaffen es von der guten Idee zur tatsächlichen klinischen Anwendung am Patienten und zur Implementierung in der täglichen Praxis. Die Gründe dafür sind vielfältiger politischer und struktureller Natur, jedoch ist der fehlende wissenschaftliche Nachweis eines Mehrnutzens häufig das Hauptproblem.

Im vorliegenden Themenheft „Innovationen in der Urologie“ präsentieren wir einen Ausschnitt aus aktuellen Forschungsprojekten der Urologie in Deutschland und versuchen deren Neuartigkeit sowie das Potenzial für einen klinischen Fortschritt darzustellen. Die thematische Ausrichtung wurde zunächst frei gestaltet, sodass unterschiedliche Perspektiven und Projektstrukturen dargestellt werden konnten. Die Beiträge beziehen sich nicht nur auf medizinisch-technische Innovationen, sondern umfassen auch Themen wie die onkologische Präzisionsmedizin und Versorgungsforschung. Darüber hinaus haben die Autoren versucht zu transportieren, wie moderne Forschungsprojekte organisiert sind und darin erfolgreiches Arbeiten möglich ist.

Derzeit werden auch politische Beweggründe für Innovationsprozesse auf nationaler und internationaler Ebene in unterschiedlichen Bereichen der Wirtschaft umfangreich diskutiert. Ohne Umgestaltungsprozesse und Neuordnungen sind kein Fortschritt und keine Nachhaltigkeit möglich. Anweisungen und Motivationsimpulse für junge Mitarbeiterinnen und Mitarbeiter medizinischer Einrichtungen fehlen häufig. Erkenntnisse aus der Forschung und kreative Lösungsansätze müssten konstruktiv umgesetzt und strukturiert begleitet werden.

Das Themenheft soll positive Anreize für all diejenigen setzen, die neben der Patientenversorgung die Urologie durch kreative Impulse und wissenschaftliche Aktivitäten weiterentwickeln möchten. Damit lassen sich erhebliche Vorteile für unsere Fachdisziplin erzielen. Zunächst geht es uns nicht darum, Forschungsergebnisse in eine ökonomisch wirksame Wertschöpfung einfließen zu lassen, sondern den „Innovationsgeist“ in unserer Fachgesellschaft aufleben zu lassen.

Die Urologie hat mehrmals bewiesen, dass sie Pionierleistungen nicht nur innerhalb und für die eigene Fachgesellschaft, sondern auch für die gesamte Medizin generieren kann. Kreative Prozesse entstehen oft aus der Not heraus, sind spontan und unkoordiniert. Es ist daher besonders schön zu sehen, dass sie in umfangreiche Forschungsvorhaben integriert werden und schlussendlich ans $\mathrm{Pa}$ tientenbett gelangen können. Trotz des Zeitalters tiefgreifender Regulierungen häufig auch Überregulierungen - vieler gesellschaftlicher Bereiche, des ökono- 
mischen Druckes im Gesundheitswesen sowie der internationalen Konkurrenz, sind zahlreiche urologische Zentren in der Lage, beeindruckende Innovationsleistungen $\mathrm{zu}$ erbringen.

Unsere Fachgesellschaft sollte nachhaltig dafür Sorge tragen, dass Innovationen, die speziell auf dem Boden beruflicher Aktivitäten entstehen, effizient in die praktische Umsetzung und Patientenversorgung überführt werden können. Komplexe Verzahnungen und Wechselwirkungen unterschiedlicher Organisationsebenen der Einrichtungen und des Gesundheitswesens dürfen keine Hindernisse sein. Daher ist es wichtig und entscheidend, eine tragbare Strategie für den Technologietransfer $\mathrm{zu}$ entwickeln, die mit den geltenden rechtlichen Bedingungen im Einklang steht. Die präsentierten Beiträge dieser Ausgabe zeigen beeindruckend, wie wichtig multi- und interdisziplinären Kooperationen zwischen Universitäten, außeruniversitären Forschungseinrichtungen und der Industrie sind und wie diese ausgestaltet werden können.

Wir hoffen, dass die Lektüre dazu inspiriert, noch mehr medizinische Innovationen und Erfindungen „aus der Schublade" der Ärzte und Forscher in den Implementierungsprozess zu überführen, um damit die Weiterentwicklung der Urologie zu ermöglichen.

Ihr

Arkadiusz Miernik, Thorsten Schlomm und Christian Bolenz

\section{Korrespondenzadresse}

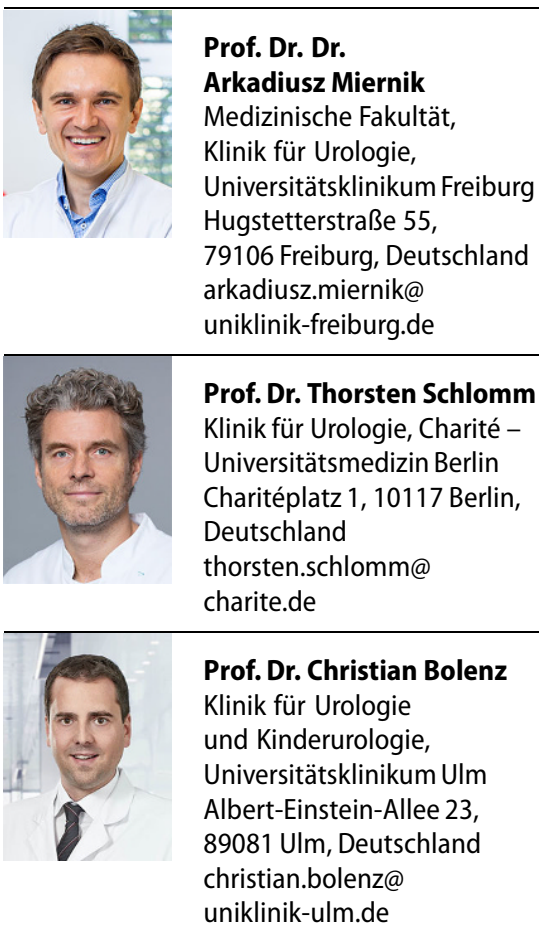

Interessenkonflikt. A. Miernik erhält Forschungsmittel durch das Bundesministerium für Bildung und Forschung (BMBF), Berlin (D). Unterstützung für seine Reisetätigkeit erfährt er durch die europäische Gesellschaft für Urologie, Arnhem (NL), sowie durch die Deutsche Gesellschaft für Urologie, Düsseldorf (D). Des Weiteren leistete A. Miernik Beratungen für: KLS Martin, Tuttlingen (D), Avatera medical, Jena (D), LISA Laser Products $\mathrm{GmbH}$, Katlenburg-Lindau (D), Schoelly fiberoptics $\mathrm{GmbH}$, Denzlingen (D), Dornier MedTech Europe GmbH (D), Medi-Tate Ltd. (IL, USA) und b.braun New ventures $\mathrm{GmbH}$, Freiburg (D). Für die Firmen RichardWolf GmbH (D) und Boston Scientific (USA) war A. Miernik als Referent tätig. Des Weiteren übte er gutachterliche Tätigkeit für die Ludwig Boltzmann Gesellschaft (A) aus. A. Miernik ist beteiligt an zahlreichen Patenten und Erfindungen im Bereich der Medizintechnik. T. Schlomm und C. Bolenz geben an, dass kein Interessenkonflikt besteht.

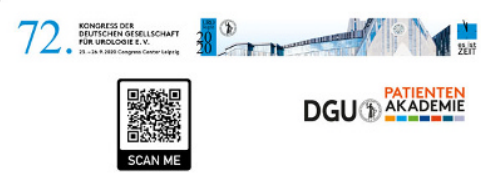

Erstes digitales

PATIENTENFORUM

ZUM 72. DGU-KONGRESS 2020

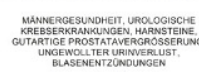

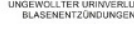

Die häufigsten urologischen

$\underset{\text { Experten }}{\text { Erkrankungen }}$

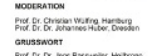

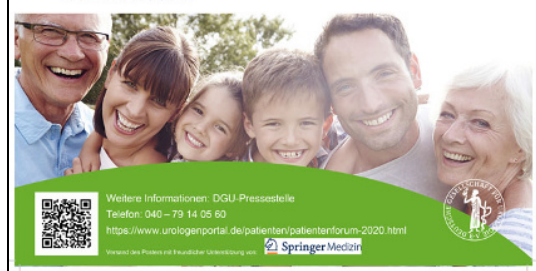

\section{Wartezimmerposter für das erste digitale DGU-Patienten- forum}

Männergesundheit, die gutartige Prostatavergrößerung, Harnsteine, ungewollter Urinverlust, Blasenentzündungen und urologische Krebserkrankungen standen im Mittelpunkt des ersten digitalen Patientenforums auf dem 72. DGU-Kongress, das jetzt allen Interessierten auf dem Urologenportal als Video-Stream zur Verfügung steht.

Deshalb bitten wir Sie, das in dieser Ausgabe des Urologen beigelegte Poster in Praxis oder Klinik aufzuhängen.

DGU-Patientenakademie 